\title{
The Impact of Statins before High-Risk CABG on Postoperative Multiple Organ Function
}

\author{
Jiayang Wang $\mathbb{D}^{1,2,3}$ Wen Yuan, ${ }^{2}$ Kui Zhang $\mathbb{D}^{1},{ }^{1}$ Nan Liu, ${ }^{2}$ Dong Liu, ${ }^{1}$ and Yujie Zhou $\mathbb{D I}^{3}$ \\ ${ }^{1}$ Department of Cardiac Surgery, Beijing An Zhen Hospital Capital Medical University, Beijing 100029, China \\ ${ }^{2}$ Center for Cardiac Intensive Care, Beijing An Zhen Hospital Capital Medical University, Beijing 100029, China \\ ${ }^{3}$ Department of Cardiology, 12th Ward, Beijing Anzhen Hospital, Beijing Institute of Heart Lung and Blood Vessel Disease, \\ Beijing Key Laboratory of Precision Medicine of Coronary Atherosclerotic Disease, Clinical Center for Coronary Heart Disease, \\ Capital Medical University, Beijing 100029, China
}

Correspondence should be addressed to Jiayang Wang; athlandwang@hotmail.com and Yujie Zhou; azzyj12@163.com

Received 5 September 2019; Accepted 6 November 2019; Published 14 January 2020

Guest Editor: Qian Fan

Copyright ( 2020 Jiayang Wang et al. This is an open access article distributed under the Creative Commons Attribution License, which permits unrestricted use, distribution, and reproduction in any medium, provided the original work is properly cited.

Background. The purpose of this cohort study was to investigate the independent relationship between preoperative statin therapy (PST) and postoperative severe multiorgan failure, measured by the Sequential Organ Failure Assessment (SOFA) maximum greater than 11, in high-risk patients undergoing isolated coronary artery bypass grafting (CABG). Methods. The present study is a perspective, single-center, cohort analysis enrolling high-risk patients undergoing CABG from Jan 1, 2018, to Dec 31, 2018, in Beijing Anzhen hospital. Results. Among a total of 880 high-risk patients undergoing isolated CABG included in this study, 503 (57.2\%) experienced statin therapy before CABG. The SOFA maximum was significantly lower in the PST group compared with the control group $(7.8 \pm 3.0 \mathrm{v} 9.2 \pm 3.4, P<0.0001)$. Multivariate logistic regression analysis demonstrated the incidence of the severe multiorgan dysfunction, measured by SOFA maximum $\geq 11$, was dramatically reduced in the PST group (OR, 0.68, 95\% CI $0.50-0.92, P=0.013$ ). Furthermore, preoperative statin therapy (PST) might be associated with a decreased risk of postoperative major adverse cardiovascular and cerebral events and acute kidney injury, but an increased risk of postoperative hepatic inadequacy. Conclusion. SOFA maximum was significantly lower in the PST group compared with the control group and the incidence of the severe multiorgan dysfunction was dramatically reduced in the PST group. The findings of this study might shed new light on questions of positive or negative effects of PST on multiple organ function after high-risk CABG, so as to ultimately improve high-risk patient in-hospital outcomes from CABG.

\section{Background}

Previous studies demonstrated that the incidence of death in coronary artery bypass grafting (CABG) ranges from 2.94 to 32.5 according to different surgical severity and population $[1,2]$. Therefore, it is essential to develop prognostic models for accurately identify mortality and morbidity after isolated CABG, especially high-risk CABG. Research data proved that the Sequential Organ Failure Assessment (SOFA) score per se as an independent risk factor for mortality after CABG and SOFA could be regarded as the most effective prognostic model for guiding the use of preventive and early therapeutic strategies to reduce mortality and morbidity for patients undergoing high-risk CABG $[2,3]$. Recently, SOFA maximum is recommended to assess multiorgan dysfunction over time and severe multiorgan failure, measured by SOFA maximum greater than 11, predicted an in-hospital mortality of $95 \%[3,4]$.

Preoperative statin therapy (PST) is known to be the most effective medications for cardiac surgical patients with hyperlipidemia [5]. However, the beneficial or detrimental effects of PST on cardiovascular and cerebral vascular, renal, respiratory and liver function in patients undergoing isolated high-risk CABG are still unclear. Results from studies that investigated the effects of PST on postoperative organ dysfunction are also controversial [6-9]. More importantly, previous studies only focused on one single organ, and no studies demonstrated the relationship between PST and 
severe multiple organ dysfunction after high-risk CABG. This may be ascribed to the lack of effective and comprehensive prediction models to evaluate postoperative multiple organ dysfunction. The appearance of SOFA maximum can solve this problem.

To fill the above knowledge gap, we systematically assessed the multiorgan function in high-risk patients undergoing isolated CABG in Beijing Anzhen hospital. The purpose of this cohort study was to investigate the independent relationship between PST and postoperative severe multiorgan failure, measured by SOFA maximum greater than 11, in high-risk patients undergoing isolated CABG and also examine the direct correlation between PST and the incidence of cardiac and cerebral vascular, respiratory, liver as well as renal postoperative complications. Recognition of the association and determinants of PST on postoperative multiple organ dysfunction should lead to strategies to improve the prognosis of patients undergoing elective high-risk CABG.

\section{Methods}

2.1. Study Population and Clinical Data. The present study is a perspective, single-center, cohort analysis enrolling highrisk patients undergoing CABG from Jan 1, 2018, to Dec 31, 2018, in Beijing Anzhen hospital. High-risk was defined as CHD patients with euroscore II of $6 \%$ or more. Patients received $20 \mathrm{mg}$ of atorvastatin per day before CABG was included in the PST group. Patients undergoing CABG combined with other open-chest surgeries such as valvular repair or replacement were excluded. The gathered data included the main baseline clinical, echocardiographic, and procedural characteristics. Two reviewers (D.L. and W.Y.) independently extracted the above information. Informed consent was obtained from each patient on the day of admission. The ethical review and informed consent of this study were approved by the institutional ethics committee of Beijing Anzhen Hospital, Capital Medical University.

2.2. Definitions and Study Endpoints. Endpoints were: (1) inhospital adverse outcomes defined as in the Society of Thoracic Surgeons (STS) national database [10]. The specific definitions are located on the STS website (http://www.sts. org/registries-research-center/sts-national-database/adultcardiac-surgery-database/data-collection). A composite endpoint of in-hospital major adverse cardiovascular and cerebral events (MACCE) and the STS-defined variables of major morbidity were utilized; (2) in-hospital SOFA maximum: sequential assessment of in-hospital organ dysfunction is a good indicator of prognosis and SOFA, which is assessed in all patients after CABG every day, can help assess organ dysfunction or failure over time and are useful to evaluate morbidity [11]. The highest in-hospital SOFA score, namely SOFA maximum, of greater than 11 predicted a mortality of $95 \%[11,12]$.

2.3. Statistical Analysis. Statistical analysis was performed using the SPSS version 25.0 statistical software (IBM
Corporation, Armonk, New York, USA). Baseline characteristics were compared between the patients with major bleeding and without major bleeding. Continuous variables were expressed as mean value \pm standard deviation and compared by the Student's $t$-test if normally distributed and otherwise as median (minimum, maximum) and compared by the Wilcoxon rank sum test. Categorical variables are expressed as percentages and were compared by the $\chi^{2}$ statistic or continuity-correction $\chi 2$ when cell counts were $<5$ or Fisher's exact test when cell counts were $<1$.

We used multivariable logistic regression analysis to investigate the association between PST and the incidence of SOFA maximum greater than 11, in-hospital postoperative MACCE, acute kidney injury (AKI), hepatic inadequacy, and infection adjusting for potential confounding factors. Forward stepwise selection was used to identify significant confounding variables. Potential confounders that had been reported in previous studies as important determinants of perioperative outcomes would be offered to the logistic regression models including: age, female gender, body mass index (BMI), diabetes, hypertension, prior MI, prior transient ischemic attacks (TIA) or cerebral vascular accident (CVA), current smoker, hypercholesterolaemia, previous peripheral vascular diseases (PVD), previous atrial fibrillation (AF), previous chronic obstructive pulmonary diseases (COPD), ventricular aneurysm, emergency CABG, decreased left ventricular ejection fraction (LVEF), euroscore II, duration of operation, off Pump CABG, New York Heart Association (NYHA) Functional Classification, and drugs before CABG. And closely associated factors $(P<0.05)$ from the univariate analysis were also included in the multivariable logistic regression analysis $(P<0.05$ was retention criterion for each factor). Power of the association between risk factors and outcomes was expressed as odds ratio (OR).

All $P$ values are 2 -sided. Results were considered to be statistically significant at a $P<0.05$.

\section{Results}

3.1. Demographic and Perioperative Characteristics. Among a total of 880 high-risk patients undergoing isolated CABG included in this study, 503 (57.2\%) experienced statin therapy before CABG. The mean time of PST was 5.2 months. Baseline, procedural, and discharge data for the patients are shown in Table 1. Compared with the control group, significantly higher proportions of patients with PST had presented with the following clinical characteristics at hospital admission: male sex $(69.2 \% \mathrm{v} 46.9 \%, P<0.0001)$, moderate and poor LVEF ( $48.1 \%$ v $40.8 \%, P=0.028)$, lower euroscore II $(8.7 \pm 4.4$ v $8.8 \pm 2.9, P=0.033)$, hypertension $(59.8 \%$ v $43.0 \%, P<0.0001)$, diabetes $(56.1 \%$ v $23.6 \%$, $P<0.0001)$, previous MI $(16.9 \%$ v7.0\%, $P<0.0001)$, preoperative angiotensin-converting enzyme inhibitors (ACEI)/angiotensin receptor blockers (ARB) $(22.3 \% \mathrm{v}$ $14.1 \%, P=0.002)$, and preoperative beta-blocker therapy (58.6\% v $41.4 \%, P<0.0001)$. On the contrary, the proportion of patients undergoing CABG without cardiopulmonary bypass was significantly lower in the PST group. Other characteristics were comparable in the two groups. 
TABLE 1: Characteristics of study population.

\begin{tabular}{|c|c|c|c|}
\hline \multirow{2}{*}{ Variable } & \multicolumn{2}{|c|}{ Preoperative statin therapy } & \multirow{2}{*}{$P$-value } \\
\hline & $\operatorname{YES}(n=503)$ & $\mathrm{NO}(n=377)$ & \\
\hline \multicolumn{4}{|l|}{ Demographics } \\
\hline Age, mean (SD), y & $65.4(7.6)$ & $64.8(10.9)$ & 0.349 \\
\hline Male sex, $n(\%)$ & $348(69.2)$ & $177(46.9)$ & $<0.0001$ \\
\hline BMI, mean (SD), $\mathrm{kg} / \mathrm{m} 2$ & $25.1(2.9)$ & $24.7(3.4)$ & 0.061 \\
\hline $\mathrm{BMI} \geq 30, n(\%)$ & $24(4.8)$ & $25(6.6)$ & 0.239 \\
\hline \multicolumn{4}{|l|}{ Medical history } \\
\hline Hypertension, $n(\%)$ & $301(59.8)$ & $162(43.0)$ & $<0.0001$ \\
\hline HLP, $n(\%)$ & $250(49.7)$ & $112(29.7)$ & $<0.0001$ \\
\hline Diabetes mellitus, $n(\%)$ & $282(56.1)$ & $89(23.6)$ & $<0.0001$ \\
\hline Smoker, $n(\%)$ & $149(29.6)$ & $91(24.1)$ & 0.079 \\
\hline COPD, $n(\%)$ & $51(10.1)$ & $35(9.3)$ & 0.731 \\
\hline PVD, $n(\%)$ & $116(23.1)$ & $72(19.1)$ & 0.116 \\
\hline Previous MI, $n(\%)$ & $85(16.9)$ & $26(7.0)$ & $<0.0001$ \\
\hline Previous CVA, $n(\%)$ & $36(7.2)$ & $18(4.8)$ & 0.158 \\
\hline Previous AF, $n(\%)$ & $68(13.6)$ & $62(16.4)$ & 0.250 \\
\hline LVEF, mean (SD), \% & $50.6(15.1)$ & $53.3(19.1)$ & 0.018 \\
\hline Moderate and poor LVEF $(<50 \%) n(\%)$ & $242(48.1)$ & $154(40.8)$ & 0.028 \\
\hline Ventricular aneurysm, $n(\%)$ & $53(10.5)$ & $25(6.6)$ & 0.055 \\
\hline \multicolumn{4}{|l|}{ Status } \\
\hline Urgent CABG, $n(\%)$ & $21(4.2)$ & $26(6.9)$ & 0.053 \\
\hline Euroscore II & $8.7(4.4)$ & $8.8(2.9)$ & 0.033 \\
\hline \multicolumn{4}{|l|}{ NYHA class, $n(\%)$} \\
\hline II & $310(61.6)$ & $255(67.6)$ & 0.076 \\
\hline III and IV & $193(38.4)$ & $122(32.4)$ & 0.076 \\
\hline Off-pump & $246(48.9)$ & $222(58.9)$ & 0.003 \\
\hline Duration of operation mean (SD), $h$ & $4.1(6.4)$ & $4.7(1.4)$ & $<0.0001$ \\
\hline \multicolumn{4}{|l|}{ Medication at discharge } \\
\hline ACEI/ARB & $112(22.3)$ & $53(14.1)$ & 0.002 \\
\hline $\mathrm{CCB}$ & $64(12.7)$ & $40(10.6)$ & 0.345 \\
\hline Aspirin & $385(76.5)$ & $274(72.7)$ & 0.209 \\
\hline Beta-blocker & $295(58.6)$ & $156(41.4)$ & $<0.0001$ \\
\hline
\end{tabular}

CABG: coronary artery bypass grafting; LVEF: left ventricular ejection fraction; BMI: body mass index; MI: myocardial infarction TIA: prior transient ischemic attacks; CVA: cerebral vascular accident; PVD: previous peripheral vascular diseases AF: previous atrial fibrillation; COPD: previous chronic obstructive pulmonary diseases; NYHA : New York Heart Association; ACEI: angiotensin-converting enzyme inhibitors; ARB: angiotensin receptor blockers; CCB: calcium channel blockers.

3.2. In-Hospital Outcomes. In-hospital outcomes for the two groups are shown in Table 2. The SOFA maximum was significantly lower in the PST group compared with the control group $(7.8 \pm 3.0$ v $9.2 \pm 3.4, P<0.0001$, Table 2$)$. In addition, the proportion of patients with SOFA maximum greater than 11 was also significantly lower in the PST group. With respect to other secondary clinical outcomes, the rate of in-hospital MACCE, especially nonfatal stroke, acute kidney injury (AKI), and noninvasive ventilator, was significantly lower in patients with PST than in controls (Table 2). On the contrary, the rate of hepatic inadequacy postinfection was higher in the PST group (Table 2).

3.3. Multivariate Logistic Regression Analysis on Severe Multiorgan Dysfunction (Primary Endpoint). Multivariate logistic regression analysis on the total patients demonstrated the incidence of the severe multiorgan dysfunction, measured by SOFA maximum $\geq 11$, was dramatically reduced in the PST group (OR, 0.68, 95\% CI 0.50-0.92, $P=0.013$, Table 3 ). On the contrary, female gender (OR, 1.93, 95\% CI 1.43-2.60, $P<0.0001$ ), higher euroscore II
(OR,1.05, 95\% CI 1.01-1.09, $P=0.012$ ), hypertension (OR, $1.40,95 \%$ CI $1.02-1.87, P=0.021$ ), previous $\mathrm{MI}(\mathrm{OR}, 1.99$, 95\% CI 1.30-3.04, $P=0.002)$, NYHA class III and IV (OR, $1.58,95 \%$ CI $1.17-2.13, P=0.003)$, moderate and poor LVEF (OR, 2.38, 95\% CI 1.76-3.21, $P<0.0001$ ), emergency CABG (OR, 5.64, 95\% CI 3.02-10.56, $P<0.0001)$, off Pump CABG (OR, 1.36, 95\% CI (1.46-1.92), $P=0.044)$, and longer duration of surgery (OR, 1.68, 95\% CI 1.46-1.92, $P<0.0001)$ (Table 3) were the independent risk factors for severe multiorgan dysfunction.

3.4. Multivariate Logistic Regression Analysis on In-Hospital MACCE. Multivariate logistic regression analysis on the total patients demonstrated PST (OR, 0.60, 95\% CI 0.44$0.81, P=0.001)$ may be associated with a decreased risk of in-hospital MACCE (Table 4). Besides, hypertension, higher euroscore II, current smoker, previous MI, previous TIA or CVA, previous AF, previous COPD, moderate and poor LVEF, NYHA III and IV, and longer duration of surgery were the independent risk factors for in-hospital MACCE (Table 4). 
TABLE 2: In-hospital outcomes.

\begin{tabular}{lccc}
\hline & PST $(n=503)$ & No PST $(n=377)$ & $P$ value \\
\hline SOFA Maximum $\geq 11$ & $24.3 \%(122 / 503)$ & $34.2 \%(129 / 377)$ & $P=0.002$ \\
SOFA maximum & $7.8(3.0)$ & $9.2(3.4)$ & $P<0.0001$ \\
MACCE & $27.6 \%(139 / 503)$ & $35.0 \%(132 / 377)$ & $P=0.022$ \\
In-hospital mortality & $1.2 \%(6 / 503)$ & $1.1 \%(4 / 377)$ & $P=1.000$ \\
$\quad$ Nonfatal MI & $4.6 \%(23 / 503)$ & $4.8 \%(18 / 377)$ & $P=1.000$ \\
$\quad$ Nonfatal stroke & $0.6 \%(3 / 503)$ & $2.9 \%(11 / 377)$ & $P=0.011$ \\
New-onset AF & $18.1 \%(91 / 503)$ & $22.8 \%(86 / 377)$ & $P=0.090$ \\
New-onset VA & $6.6 \%(33 / 503)$ & $11.4 \%(43 / 377)$ & $P=0.015$ \\
Perioperative IABP & $13.3 \%(67 / 503)$ & $11.9 \%(45 / 377)$ & $P=0.610$ \\
Perioperative ECMO & $0.6 \%(3 / 503)$ & $0.0 \%(0 / 377)$ & $P=0.265$ \\
Reoperation & $3.6 \%(18 / 503)$ & $1.9 \%(7 / 377)$ & $P=0.153$ \\
After infection & $17.3 \%(87 / 503)$ & $9.0 \%(34 / 377)$ & $P<0.0001$ \\
Pulmonary infection & $16.1 \%(81 / 503)$ & $9.0 \%(34 / 377)$ & $P=0.002$ \\
After bloodstream infection & $1.2 \%(6 / 503)$ & $0.0 \%(0 / 377)$ & $P=0.040$ \\
AKI & $5.4 \%(27 / 503)$ & $2.7 \%(10 / 377)$ & $P<0.0001$ \\
CRRT & $2.8 \%(14 / 503)$ & $1.3 \%(5 / 377)$ & $P=0.165$ \\
Hepatic inadequacy & $17.7 \%(89 / 503)$ & $12.5 \%(47 / 377)$ & $P=0.047$ \\
Hypoxemia & $8.5 \%(43 / 503)$ & $6.1 \%(23 / 377)$ & $P=0.197$ \\
Noninvasive ventilator & $2.0 \%(10 / 503)$ & $8.0 \%(30 / 377)$ & $P<0.0001$ \\
Reintubation & $2.2 \%(11 / 503)$ & $2.4 \%(9 / 377)$ & $P=1.000$ \\
Tracheotomy & $1.0 \%(5 / 503)$ & $0.0 \%(0 / 377)$ & $P=0.075$ \\
ICU stay (Day) & $2.7(3.7)$ & $2.4(1.8)$ & $P .0(3.0)$ \\
Postoperative hospital stay (Day) & $8.1(5.4)$ & $137,345.6(36,636.8)$ & \\
Cost (RMB) & $138,636.1(62,142.1)$ & $P=0.839$ \\
\hline
\end{tabular}

MACCE: major adverse cardiovascular and cerebral events; MI: myocardial infarction; AF: previous atrial fibrillation; AKI: acute kidney injury; CRRT: continuous renal replacement therapy; IABP: intra-aortic balloon pump; ECMO: extracorporeal membrane oxygenation; SOFA: Sequential Organ Failure Assessment; VA: ventricular arrhythmias; PST: preoperative statin therapy.

TABLE 3: Independent risk factors for SOFA maximum greater than 11.

\begin{tabular}{|c|c|c|c|c|c|c|}
\hline & \multicolumn{2}{|c|}{ Maximum SOFA score >11 } & \multirow{2}{*}{$X^{2} / t$} & \multirow{2}{*}{$P$ value } & \multirow{2}{*}{ Multivariate analysis OR (95\% CI) } & \multirow{2}{*}{$P$ value } \\
\hline & YES $(n=251)$ & NO $(n=629)$ & & & & \\
\hline PST & $122(48.6)$ & $381(60.6)$ & 10.5 & 0.013 & $0.68(0.50-0.92)$ & 0.013 \\
\hline ACE inhibitor or ARB & $56(22.3)$ & $109(17.3)$ & 2.9 & 0.104 & & \\
\hline Advanced age & $66.0 \pm 7.7$ & $64.8 \pm 9.6$ & 1.8 & 0.070 & & \\
\hline Female gender & $130(51.8)$ & $225(35.8)$ & 19.1 & $<0.0001$ & $1.93(1.43-2.60)$ & $<0.0001$ \\
\hline Euroscore II & $9.5 \pm 3.8$ & $8.5 \pm 3.8$ & 3.5 & $<0.0001$ & $1.05(1.01-1.09)$ & 0.012 \\
\hline BMI & $24.8 \pm 3.2$ & $25.0 \pm 3.1$ & 0.63 & 0.531 & & \\
\hline Hypertension & $149(59.4)$ & $314(49.9)$ & 6.4 & 0.014 & $1.40(1.02-1.87)$ & 0.021 \\
\hline Current smoker & $61(24.39)$ & $179(28.5)$ & 1.6 & 0.241 & & \\
\hline Previous MI & $46(18.3)$ & $65(10.3)$ & 10.4 & 0.002 & $1.99(1.30-3.04)$ & 0.002 \\
\hline Previous TIA or CVA & $9(3.6)$ & $45(7.2)$ & 3.9 & 0.061 & & \\
\hline Hypercholesterolaemia & $115(45.8)$ & $247(39.3)$ & 3.2 & 0.081 & & \\
\hline Previous AF & $39(15.5)$ & $91(10.3)$ & 0.16 & 0.675 & & \\
\hline Previous COPD & $17(6.8)$ & $69(11.0)$ & 3.6 & 0.060 & & \\
\hline Ventricular aneurysm & $20(8.0)$ & $51(8.1)$ & 0.35 & 0.601 & & \\
\hline Moderate and poor LVEF $(<50 \%)$ & $152(60.6)$ & $244(38.8)$ & 33.5 & $<0.0001$ & $2.38(1.76-3.21)$ & $<0.0001$ \\
\hline NYHA III and IV & $109(43.4)$ & $206(32.8)$ & 8.90 & 0.003 & $1.58(1.17-2.13)$ & 0.003 \\
\hline Emergency CABG & $31(12.4)$ & $16(2.5)$ & 34.1 & $<0.0001$ & $5.64(3.02-10.56)$ & $<0.0001$ \\
\hline Off pump & $147(58.6)$ & $321(51.0)$ & 4.1 & 0.044 & $1.36(1.01-1.82)$ & 0.044 \\
\hline Duration of operation (hours) & $4.8 \pm 1.5$ & $4.2 \pm 0.8$ & 157.2 & $<0.0001$ & $1.68(1.46-1.92)$ & $<0.0001$ \\
\hline
\end{tabular}

PST: preoperative statin therapy; CABG: coronary artery bypass grafting; LVEF: left ventricular ejection fraction; BMI: body mass index; MI: myocardial infarction; TIA: prior transient ischemic attacks; CVA: cerebral vascular accident; PVD: previous peripheral vascular diseases; DM: diabetes mellitus; NYHA : New York Heart Association; ACEI: angiotensin-converting enzyme inhibitors; ARB: angiotensin receptor blockers.

3.5. Multivariate Logistic Regression Analysis on AKI. Multivariate logistic regression analysis on the total patients demonstrated that PST (OR, 0.25, 95\% CI 0.12-0.54, $P<0.0001)$ and ACE inhibitor or ARB may be associated with a decreased risk of postoperative AKI (Table 5). Besides, current smoker, emergency CABG, and longer duration of surgery were the independent risk factors for postoperative AKI (Table 5). 
TABLE 4: Independent risk factors for in-hospital MACCE.

\begin{tabular}{lccccc}
\hline & \multicolumn{2}{c}{ In-hospital MACCE } & \multirow{2}{*}{$X^{2} / t$} & $P$ value & Multivariate analysis OR (95\% CI) $P$ value \\
& YES $(n=271)$ & NO $(n=609)$ & & $0.60(0.44-0.81)$ \\
PST & $139(51.3)$ & $364(59.8)$ & 5.5 & 0.022 & 0.001 \\
ACE inhibitor or ARB & $48(17.7)$ & $117(19.2)$ & 0.3 & 0.641 & \\
Female gender & $117(43.2)$ & $238(39.1)$ & 1.3 & 0.265 & $1.04(1.01-1.08)$ \\
Euroscore II & $9.2 \pm 5.0$ & $8.6 \pm 3.2$ & 26.7 & $<0.0001$ & \\
BMI & $25.5 \pm 3.8$ & $24.7 \pm 3.7$ & 1.7 & 0.193 & $2.41(1.78-3.28)$ \\
Hypertension & $178(65.7)$ & $285(46.8)$ & 26.8 & $<0.0001$ & \\
DM & $111(41.0)$ & $260(42.7)$ & 0.2 & 0.658 & $1.60(1.16-2.21)$ \\
Current smoker & $96(34.7)$ & $144(23.6)$ & 13.1 & $<0.0001$ & $1.57(1.03-2.40)$ \\
Previous MI & $45(16.6)$ & $66(10.8)$ & 5.7 & 0.021 & $2.81(1.60-4.93)$ \\
Previous TIA or CVA & $29(10.7)$ & $25(4.1)$ & 14.2 & $<0.0001$ & $<0.0001$ \\
Previous PVD & $67(24.7)$ & $124(20.4)$ & 2.1 & 0.157 & 0.004 \\
Hypercholesterolaemia & $110(40.1)$ & $252(41.2)$ & 0.05 & 0.882 & 0.035 \\
Previous AF & $55(20.3)$ & $75(12.3)$ & 9.5 & 0.003 & $1.67(1.12-2.45)$ \\
Previous COPD & $38(14.0)$ & $48(7.9)$ & 8.0 & 0.001 & $2.28(1.42-3.66)$ \\
Ventricular aneurysm & $19(7.0)$ & $59(9.7)$ & 1.7 & 0.247 & \\
Moderate and poor LVEF (<50\%) & $146(53.9)$ & $250(41.1)$ & 12.4 & 0.001 & $1.79(1.33-2.42)$ \\
NYHA III and IV & $115(42.4)$ & $200(32.8)$ & 7.5 & 0.008 & $1.57(1.17-2.11)$ \\
Emergency CABG & $18(6.6)$ & $29(4.8)$ & 1.3 & 0.258 & 0.0001 \\
Off pump & $137(56.5)$ & $331(49.8)$ & 1.1 & 0.306 & 0.011 \\
Duration of operation (hours) & $4.5 \pm 1.3$ & $4.3 \pm 1.0$ & 1.1 & 0.003 & $<0.0001$ \\
\hline
\end{tabular}

PST: preoperative statin therapy; CABG: coronary artery bypass grafting; MACCE: major adverse cardiovascular and cerebral events; LVEF: left ventricular ejection fraction; BMI: body mass index; MI: myocardial infarction; TIA: prior transient ischemic attacks; CVA: cerebral vascular accident; PVD: previous peripheral vascular diseases; DM: diabetes mellitus; NYHA:New York Heart Association, ACEI: angiotensin-converting enzyme inhibitors; ARB: angiotensin receptor blockers.

TABLE 5: Independent risk factors for AKI.

\begin{tabular}{lccccc}
\hline & \multicolumn{2}{c}{ AKI } & \multirow{2}{*}{$X^{2} / t$} & \multirow{2}{*}{$P$ value } & Multivariate analysis OR (95\% CI) $P$ value \\
& YES $(n=37)$ & NO $(n=843)$ & & & $0.25(0.12-0.54)$ \\
\hline PST & $10(27.0)$ & $493(58.5)$ & 14.3 & $<0.0001$ & $0.14(0.02-1.01)$ \\
ACE inhibitor or ARB & $1(2.7)$ & $164(19.5)$ & 6.5 & 0.008 & 0.0001 \\
Female gender & $17(45.9)$ & $338(40.1)$ & 0.5 & 0.497 & \\
Euroscore II & $7.8 \pm 1.2$ & $8.8 \pm 3.9$ & 17.7 & 0.112 & \\
BMI & $25.5 \pm 3.3$ & $24.9 \pm 3.1$ & 0.05 & 0.247 & \\
Hypertension & $24(64.9)$ & $439(52.1)$ & 2.3 & 0.134 & \\
Current smoker & $25(67.6)$ & $215(25.5)$ & 31.6 & $<0.0001$ & $(3.40-14.34)$ \\
Previous MI & $5(13.5)$ & $106(12.6)$ & 0.03 & 0.801 & \\
Previous TIA or CVA & $0(0.0)$ & $54(6.4)$ & 2.5 & 0.161 & \\
Previous PVD & $8(21.6)$ & $183(21.7)$ & 0.01 & 1.000 & \\
Previous AF & $7(20.3)$ & $123(12.3)$ & 0.5 & 0.476 & \\
Previous COPD & $5(13.5)$ & $81(9.6)$ & 0.6 & 0.397 & \\
Ventricular aneurysm & $19(7.0)$ & $59(9.7)$ & 1.7 & 0.247 & \\
Moderate and poor LVEF $(<50 \%)$ & $15(53.9)$ & $381(41.1)$ & 0.3 & 0.615 & \\
Emergency CABG & $6(16.2)$ & $41(4.9)$ & 9.0 & 0.011 & $3.79(1.50-9.58)$ \\
Off pump & $24(64.9)$ & $444(52.7)$ & 2.1 & 0.178 & \\
Duration of operation (hours) & $6.9 \pm 1.7$ & $4.2 \pm 0.9$ & 44.5 & $<0.0001$ & $4.47(3.27-6.12)$ \\
\hline
\end{tabular}

PST: preoperative statin therapy; CABG: coronary artery bypass grafting; LVEF: left ventricular ejection fraction; BMI: body mass index; MI: myocardial infarction; TIA: prior transient ischemic attacks; CVA: cerebral vascular accident; PVD: previous peripheral vascular diseases; DM: diabetes mellitus; NYHA : New York Heart Association; ACEI: angiotensin-converting enzyme inhibitors; ARB: angiotensin receptor blockers.

3.6. Multivariate Logistic Regression Analysis on In-Hospital Hepatic Inadequacy. Multivariate logistic regression analysis on the total patients revealed that the independent risk factors for in-hospital hepatic inadequacy were PST (OR, 1.49, 95\% CI 1.01-2.18, $P=0.042$ ), hypertension, previous $\mathrm{MI}$, previous PVD, and previous COPD. Besides, female gender and off Pump CABG may be associated with a decreased risk of in-hospital hepatic inadequacy (Table 6).

3.7. Independent Risk Factors for Postoperative Infection. Multivariate logistic regression analysis on the total patients revealed that the independent risk factors for postoperative 
TAвLE 6: Independent risk factors for In-hospital hepatic inadequacy.

\begin{tabular}{|c|c|c|c|c|c|c|}
\hline & \multicolumn{2}{|c|}{ In-hospital hepatic inadequacy } & \multirow{2}{*}{$X^{2} / t$} & \multirow{2}{*}{$P$ value } & \multirow{2}{*}{ Multivariate analysis OR (95\% CI) } & \multirow{2}{*}{$P$ value } \\
\hline & YES $(n=136)$ & NO $(n=739)$ & & & & \\
\hline PST & $89(65.4)$ & $414(56.3)$ & 4.2 & 0.047 & $1.49(1.01-2.18)$ & 0.042 \\
\hline Female gender & $35(25.7)$ & $315(42.6)$ & 13.7 & $<0.0001$ & $0.47(0.31-0.72)$ & $<0.0001$ \\
\hline Euroscore II & $9.4 \pm 5.4$ & $9.0 \pm 3.5$ & 2.0 & 0.050 & & \\
\hline BMI & $24.2 \pm 2.9$ & $25.0 \pm 3.1$ & -2.5 & 0.357 & & \\
\hline Hypertension & $93(68.4)$ & $365(49.4)$ & 16.6 & $<0.0001$ & $2.18(1.48-3.23)$ & $<0.0001$ \\
\hline Current smoker & $40(29.4)$ & $200(27.1)$ & 0.3 & 0.601 & & \\
\hline Previous MI & $25(18.4)$ & $86(11.6)$ & 4.7 & 0.035 & $1.72(1.05-2.80)$ & 0.030 \\
\hline Previous TIA or CVA & $8(5.9)$ & $46(6.0)$ & 0.02 & 1.000 & & \\
\hline Previous PVD & $40(29.4)$ & $151(20.4)$ & 5.4 & 0.024 & $1.63(1.08-2.46)$ & 0.020 \\
\hline Previous COPD & $34(25.0)$ & $47(6.9)$ & 47.5 & $<0.0001$ & $4.09(2.47-6.78)$ & $<0.0001$ \\
\hline Ventricular aneurysm & $7(5.1)$ & $71(9.6)$ & 2.8 & 0.103 & & \\
\hline NYHA III and IV & $51(37.5)$ & $264(35.7)$ & 0.2 & 0.698 & & \\
\hline Emergency CABG & $5(3.7)$ & $42(5.7)$ & 0.9 & 0.413 & & \\
\hline Off pump & $51(37.5)$ & $415(56.2)$ & 16.1 & $<0.0001$ & $0.59(0.40-0.87)$ & 0.008 \\
\hline Duration of operation (hours) & $4.5 \pm 1.4$ & $4.3 \pm 1.0$ & 1.8 & 0.079 & & \\
\hline
\end{tabular}

PST : Preoperative statin therapy; CABG: coronary artery bypass grafting; LVEF: left ventricular ejection fraction; BMI: body mass index; MI: myocardial infarction; TIA: prior transient ischemic attacks; CVA: cerebral vascular accident; PVD: previous peripheral vascular diseases; DM: diabetes mellitus COPD: previous chronic obstructive pulmonary diseases; NYHA : New York Heart Association.

TABLE 7: Independent risk factors for postoperative infection.

\begin{tabular}{lccccc}
\hline & \multicolumn{2}{c}{ Postoperative infection } & \multirow{2}{*}{$X^{2} / t$} & \multirow{2}{*}{$P$ value } & Multivariate analysis OR (95\% CI) $P$ value \\
& YES $(n=121)$ & NO $(n=759)$ & & & $2.09(1.42-3.08)$ \\
PST & $87(71.9)$ & $416(54.8)$ & 12.5 & $<0.0001$ & $<0.0001$ \\
Female gender & $42(34.7)$ & $313(41.2)$ & 1.8 & 0.195 & \\
Euroscore II & $8.4 \pm 3.9$ & $8.8 \pm 3.8$ & -1.1 & 0.275 & $1.07(1.01-1.14)$ \\
BMI & $25.5 \pm 3.3$ & $24.8 \pm 3.1$ & 2.3 & 0.024 & \\
Hypertension & $68(56.2)$ & $395(52.0)$ & 0.7 & 0.433 & $1.76(1.17-2.65)$ \\
DM & $70(57.9)$ & $301(39.7)$ & 14.2 & $<0.0001$ & $1.73(1.03-2.89)$ \\
Previous MI & $26(21.5)$ & $85(11.2)$ & 10.0 & 0.003 & 0.024 \\
Previous TIA or CVA & $11(9.1)$ & $43(5.7)$ & 2.1 & 0.153 & 0.038 \\
Previous PVD & $22(18.2)$ & $169(22.3)$ & 1.0 & 0.344 & \\
Hypercholesterolaemia & $43(35.5)$ & $319(42.0)$ & 1.8 & 0.196 & $4.44(2.65-7.43)$ \\
Previous AF & $18(14.9)$ & $112(14.8)$ & 0.001 & 1.000 & $1.82(1.22-2.72)$ \\
Previous COPD & $6(5.0)$ & $80(10.5)$ & 3.7 & 0.068 & $2.60(1.75-3.86)$ \\
Ventricular aneurysm & $30(24.8)$ & $48(6.3)$ & 44.1 & $<0.0001$ & $2.02(1.01-4.04)$ \\
Moderate and poor LVEF $(<50 \%)$ & $72(59.5)$ & $324(42.3)$ & 11.6 & 0.001 & $<0.0001$ \\
NYHA III and IV & $69(57.0)$ & $256(33.7)$ & 27.5 & $<0.0001$ & $<.003$ \\
Emergency CABG & $13(10.7)$ & $34(44.8)$ & 8.1 & 0.008 & 0.0001 \\
Off pump & $63(52.1)$ & $405(53.4)$ & 0.07 & 0.845 & 0.047 \\
Duration of operation (hours) & $4.2 \pm 1.0$ & $4.4 \pm 1.1$ & -1.1 & 0.305 & \\
\hline PST &
\end{tabular}

PST: preoperative statin therapy; CABG: coronary artery bypass grafting; LVEF: left ventricular ejection fraction; BMI: body mass index; MI: myocardial infarction; TIA: prior transient ischemic attacks; CVA: cerebral vascular accident; PVD: previous peripheral vascular diseases; DM: diabetes mellitus; NYHA : New York Heart Association.

infection were PST (OR, 2.09, 95\% CI 1.42-3.08, $P<0.0001)$, $\mathrm{BMI}, \mathrm{DM}$, previous MI, a history of ventricular aneurysm, moderate and poor LVEF, NYHA III and IV, and emergency CABG (Table 7).

\section{Discussion}

This is the first study to prospectively explore the independent association between PST and postoperative severe multiorgan failure, measured by SOFA maximum greater than 11, in high-risk patients undergoing isolated CABG. Besides, we also examined the direct correlation between PST and the incidence of cardiac and cerebral vascular, respiratory, liver as well as renal postoperative complications. Our key findings are: (1) the SOFA maximum was significantly lower in the PST group compared with the control group and multivariate logistic regression analysis on the total patients demonstrated the incidence of the severe multiorgan dysfunction, measured by SOFA maximum $\geq 11$, was dramatically reduced in the PST group; (2) PST might be associated with a decreased risk of postoperative MACCE and AKI, but an increased risk of postoperative hepatic inadequacy. Respiratory complications, such as hypoxemia, reintubation, and tracheotomy, 
were comparable in the PST and control groups; (3) PST was also independently associated with postinfection.

Although statin is used in a large proportion of patients before surgery, its potential impact on postoperative multiorgan function is still incompletely understood. With respect to renal function, Singh et al. found that PST reduced the CRRT and cardiac mortality significantly but exerted no effects on the incidence of AKI after CABG. [6] Wang et al. also demonstrated that PST may not reduce the risk of AKI in patients following isolated CABG. [13] On the contrary, Layton found that statin therapy immediately before CABG may modestly reduce the incidence of postoperative AKI, particularly in younger CABG patients. [14] Our previous evidence-based study including 59,771 patients also confirmed that PST significantly reduced the risk for postoperative AKI regardless of the types of diagnosis and staging criteria in cardiac surgical patients. [7] In addition, the cardiovascular protective effects of PST have already been well recognized. Knatterud et al. proved that PST delayed the progression of atherosclerosis and further reduced the risk for postoperative cardiovascular events in coronary heart disease patients following revascularization. [15] Furthermore, the uncertain safety of statin on liver function in high-risk CABG remains a major concern, and studies on statin-induced hepatotoxicity after high-risk CABG are sparse. A review preliminarily revealed the hepatotoxicity of statins and other lipid-lowering drugs. [16] They demonstrated that both simvastatin and atorvastatin have been correlated with more than 50 case reports of liver dysfunction and other statins have been implicated in this type of liver dysfunction as well. Another research found the association between dose escalation of atorvastatin and acute liver failure. [17] However, the adverse effects of PST on liver outcomes among cardiac surgical populations still need to be investigated further. Besides, respiratory complications after CABG are common, with an occurrence of 10 to $25 \%$ [18]. However, the independent relationship between PST and respiratory complications has not been confirmed because of a paucity of data [8]. Relevant high-quality prospective studies are still essential. Last but not least, neurologic complications, especially stroke, are associated with increased mortality and longer hospitalization [19]. However, previous research found that PST was not associated with a decreased risk for stroke and encephalopathy after high-risk CABG $[20,21]$. The current research demonstrated that PST might be associated with a decreased risk of postoperative MACCE and AKI, but an increased risk of postoperative hepatic inadequacy. Respiratory complications, such as hypoxemia, reintubation, and tracheotomy, were comparable in the PST and control groups. More importantly, the incidence of the severe multiorgan dysfunction, measured by SOFA maximum $\geq 11$, was dramatically reduced in the PST group compared with the controls.

Based on the above knowledge, PST has exerted a positive effect on cardiac, neurological, and renal function, and a negative effect on liver function after high-risk CABG. However, the association between PST and postoperative severe multiple organ dysfunction is still unknown. The current study first demonstrated that SOFA maximum was significantly lower in the PST group compared with the control group and the incidence of the severe multiorgan dysfunction, measured by SOFA maximum $\geq 11$, was dramatically reduced in the PST group. The benefits of PST for cardiac dysfunction and mortality after high-risk CABG have been well established [8]. Recently, researchers have demonstrated that PST may benefit not only cardiac but also renal, neurological, and respiratory function. The pathophysiological mechanisms underlying the positive effects of PST on neurological, respiratory, and renal function might be closely related to the non-lipid-lowering activities of statins [22]. First of all, inflammation during CABG is reported to be a potential cause of organ dysfunction [23]. Previous studies proved PST could increase the release of anti-inflammation cytokines and reduce the levels of proinflammatory mediators, such as interleukin-6, interleukin- 8 , and tumor necrosis factor- $\alpha[9,14]$. In addition, ischemia-reperfusion injury as well as endothelial dysfunction are reported to be both independent associated with an increased risk of multiple organ dysfunction in patients undergoing high-risk CABG, especially on-pump surgery [24]. The pleiotropic effects of PST also include improvement in endothelial function and attenuation of reperfusion injury, which can decrease the risk of multiple organ dysfunction directly after CABG and further improve the prognosis of surgery $[25,26]$. Recently, a high-quality prospective report confirmed that preoperative high-dose atorvastatin therapy could protect myocardium in patients following coronary revascularization by decreasing the risk of ischemia-reperfusion injury and endothelial damage during surgery [27]. Besides, the beneficial impacts of PST on multiple organ dysfunction might also be attributed to the following activity of statins: antithrombosis [7]. The above positive effects of PST on multiple organ function outweigh its side effects on liver function, leading to the incidence of the severe multiorgan dysfunction, measured by SOFA maximum $\geq 11$, which was dramatically reduced in the PST group.

It is worth mentioning that the current study found that respiratory complications, such as hypoxemia, reintubation, and tracheotomy, were comparable in the PST and control groups. This might be due to the beneficial impacts of PST on respiratory dysfunction that cannot offset other etiologiesinduced respiratory dysfunction [28]. Specifically, all of the prolonged mechanical ventilation, hypoxemia, reintubation, and tracheotomy might be regarded as a clinical endpoint of multiconditions including those that have no connection with the beneficial impacts of PST (lipid-lowering activities, anti-inflammation, antithrombosis, and improvement in endothelial function.), such as a prolonged residual anesthesia effect, a major or life-threatening bleeding as well as atelectasis. Those etiologies could offset the organ protective impacts of PST and may make the benefit of PST on respiratory function less noticeable than on a cardiac, neurological, and renal function $[29,30]$.

This study has several limitations. Firstly, the present study is a perspective, single-center, cohort analysis. Highquality, large-scale, and multicenter randomized controlled trials are required to further confirm the conclusion. 
Secondly, although SOFA maximum is recommended to assess multiorgan dysfunction over time and severe multiorgan failure, measured by SOFA maximum greater than 11, predicted an in-hospital mortality of $95 \%$, we still need other organ failure assessments to measure severe multiorgan failure in order to verify each other. Thirdly, we confirmed that PST increase was associated with an increased risk of other endpoints, such as noninvasive ventilator. However, the independence of the correlation still needs further verification. Last but not least, the impact of PST on the longterm multiple organ function requires further examination.

\section{Conclusions}

This current observational cohort analysis demonstrated that PST might be associated with a decreased risk of postoperative MACCE and AKI, but an increased risk of postoperative hepatic inadequacy. Respiratory complications were comparable in the PST and control groups. In addition, SOFA maximum was significantly lower in the PST group compared with the control group, and multivariate logistic regression analysis on the total patients demonstrated that the incidence of the severe multiorgan dysfunction, measured by SOFA maximum $\geq 11$, was dramatically reduced in the PST group. The findings of this study might shed new light on questions of positive or negative effects of PST on multiple organ function after highrisk $C A B G$, and can ultimately improve high-risk patient inhospital outcomes from CABG.

\section{Abbreviations}

PST: $\quad$ Preoperative statin therapy

CABG: Coronary artery bypass grafting

CHD: Coronary heart disease

MACCE: Major adverse cardiovascular and cerebral events

SOFA: Sequential organ failure assessment

LVEF: Left ventricular ejection fraction

BMI: $\quad$ Body mass index

MI: $\quad$ Myocardial infarction

TIA: Prior transient ischemic attacks

CVA: Cerebral vascular accident

PVD: $\quad$ Previous peripheral vascular diseases

AF: $\quad$ Previous atrial fibrillation

COPD: Previous chronic obstructive pulmonary diseases

NYHA: New York Heart Association

AKI: Acute kidney injury

CRRT: Continuous renal replacement therapy

IABP: Intra-aortic balloon pump

ECMO: Extracorporeal membrane oxygenation

ACEI: Angiotensin-converting enzyme inhibitors

ARB: Angiotensin receptor blockers.

\section{Data Availability}

No data were used to support this study.

\section{Conflicts of Interest}

The authors declare that they have no conflicts of interest.

\section{Authors' Contributions}

Dr. Jiayang Wang and Prof. Yujie Zhou designed the current study, and drafted the manuscript. Dr. Wen Yuan and Dr. Dong Liu independently extracted the information from the eligible studies. Besides, Dr. Kui Zhang performed the statistical analysis. Dr. Nan Liu participated in the quality assessment.

\section{Acknowledgments}

This study was supported by the Foundation of Beijing Anzhen Hospital, Capital Medical University (No. 2016Z01), the National Natural Science Foundation of China (Grant No. 81900098), the "Beijing Municipal Administration of Hospitals" Youth Plan (Code: QML20190601), the talents support program of Organization Department of Beijing Municipal Committee (No. 2017000021469G221), the program of the Beijing Municipal Administration of Hospitals (No. XMLX201822), National Science and Technology Support Program of China (No. 2015BAI12B03), the Special Research Fund for Public Welfare Industry of Health from National Health and Family planning Commission of China (No. 201402009), National Key Research and Development Program of China (2017YFC0908800), the Beijing Municipal Administration of Hospitals Clinical Medicine Development of Special Funding Support (code: ZYLX201303), the National Key Clinical Speciality Construction Project (2013-2014), and the "Beijing Municipal Administration of Hospitals" Ascent Plan (Code: DFL20150601).

\section{References}

[1] S. Siregar, R. H. H. Groenwold, B. A. J. M. de Mol et al., "Evaluation of cardiac surgery mortality rates: 30-day mortality or longer follow-up?," European Journal of CardioThoracic Surgery, vol. 44, no. 5, pp. 875-883, 2013.

[2] C. H. Chang, S. W. Chen, P. C. Fan et al., "Sequential organ failure assessment score predicts mortality after coronary artery bypass grafting," BMC Surg, vol. 17, no. 1, p. 22, 2017.

[3] J. Wang, W. Yuan, R. Dong, N. Liu, D. Liu, and Y. Zhou, "Predictors for euthyroid sick syndrome and its impact on inhospital clinical outcomes in high-risk patients undergoing coronary artery bypass grafting," Perfusion, vol. 34, no. 8, pp. 679-688, 2019.

[4] M. Antonelli, R. Moreno, J. L. Vincent et al., "Application of SOFA score to trauma patients," Intensive Care Medicine, vol. 25, no. 4, pp. 389-394, 1999.

[5] J. L. Huffmyer, W. J. Mauermann, R. H. Thiele, J. Z. Ma, and E. C. Nemergut, "Preoperative statin administration is associated with lower mortality and decreased need for postoperative hemodialysis in patients undergoing coronary artery bypass graft surgery," Journal of Cardiothoracic and Vascular Anesthesia, vol. 23, no. 4, pp. 468-473, 2009.

[6] I. Singh, S. Rajagopalan, A. Srinivasan et al., "Preoperative statin therapy is associated with lower requirement of renal replacement therapy in patients undergoing cardiac surgery: a meta-analysis of observational studies," Interactive CardioVascular and Thoracic Surgery, vol. 17, no. 2, pp. 345-352, 2013.

[7] J. Wang, C. Gu, M. Gao, W. Yu, and Y. Yu, "Preoperative statin therapy and renal outcomes after cardiac surgery: a 
meta-analysis and meta-regression of 59,771 patients," $\mathrm{Ca}$ nadian Journal of Cardiology, vol. 31, no. 8, pp. 1051-1060, 2015.

[8] R. Komatsu, H. O. Yilmaz, J. You et al., "Lack of association between preoperative statin use and respiratory and neurologic complications after cardiac surgery," Anesthesiology, vol. 126, no. 5, pp. 799-809, 2017.

[9] J. Wang, C. Gu, M. Gao, W. Yu, and Y. Yu, "Preoperative statin therapy is associated with reduced 30 -day postoperative all-cause mortality in patients undergoing coronary artery bypass surgery: a meta-analysis of large size observational studies," International Journal of Cardiology, vol. 181, pp. 11-13, 2015.

[10] S. Raza, J. F. Sabik III, P. Ainkaran, and E. H. Blackstone, "Coronary artery bypass grafting in diabetics: a growing health care cost crisis," The Journal of Thoracic and Cardiovascular Surgery, vol. 150, no. 2, pp. 304-312, 2015.

[11] V. Pettila, "Sequential assessment of multiple organ dysfunction as a predictor of outcome," JAMA: The Journal of the American Medical Association, vol. 287, no. 6, pp. 713-714, 2002.

[12] F. L. Ferreira, D. P. Bota, A. Bross, C. Melot, and J. L. Vincent, "Serial evaluation of the SOFA score to predict outcome in critically ill patients," JAMA, vol. 286, no. 14, pp. 1754-1758, 2001.

[13] Y. Wang, S. Zhu, R. Du, J. Zhou, Y. Chen, and Q. Zhang, "Statin initiation and renal outcomes following isolated coronary artery bypass grafting: a meta-analysis," The Journal of Cardiovascular Surgery, vol. 59, no. 2, pp. 282-290, 2018.

[14] J. B. Layton, A. V. Kshirsagar, R. J. Simpson Jr. et al., "Effect of statin use on acute kidney injury risk following coronary artery bypass grafting," The American Journal of Cardiology, vol. 111, no. 6, pp. 823-828, 2013.

[15] G. L. Knatterud, Y. Rosenberg, L. Campeau et al., "Long-term effects on clinical outcomes of aggressive lowering of lowdensity lipoprotein cholesterol levels and low-dose anticoagulation in the post coronary artery bypass graft trial," Circulation, vol. 102, no. 2, pp. 157-165, 2000.

[16] E. S. Björnsson, "Hepatotoxicity of statins and other lipidlowering agents," Liver International, vol. 37, no. 2, pp. 173-178, 2017.

[17] M. F. Carrascosa, J. R. Salcines-Caviedes, M. I. Lucena, and R. J. Andrade, "Acute liver failure following atorvastatin dose escalation: is there a threshold dose for idiosyncratic hepatotoxicity?," Journal of Hepatology, vol. 62, no. 3, pp. 751-752, 2015.

[18] J. F. H. Ubben, M. D. Lance, W. F. Buhre, and J. U. Schreiber, "Clinical strategies to prevent pulmonary complications in cardiac surgery: an overview," Journal of Cardiothoracic and Vascular Anesthesia, vol. 29, no. 2, pp. 481-490, 2015.

[19] G. W. Roach, M. Kanchuger, C. M. Mangano et al., "Adverse cerebral outcomes after coronary bypass surgery," New England Journal of Medicine, vol. 335, no. 25, pp. 1857-1864, 1996.

[20] M. A. Koenig, M. A. Grega, M. M. Bailey et al., "Statin use and neurologic morbidity after coronary artery bypass grafting: a cohort study," Neurology, vol. 73, no. 24, pp. 2099-2106, 2009.

[21] I. S. Ali and K. J. Buth, "Preoperative statin use and outcomes following cardiac surgery," International Journal of Cardiology, vol. 103, no. 1, pp. 12-18, 2005.

[22] Z. A. Massy, W. F. Keane, and B. L. Kasiske, "Inhibition of the mevalonate pathway: benefits beyond cholesterol reduction?," The Lancet, vol. 347, no. 8994, pp. 102-103, 1996.
[23] A. Sauaia, F. A. Moore, and E. E. Moore, "Postinjury inflammation and organ dysfunction," Critical Care Clinics, vol. 33, no. 1, pp. 167-191, 2017.

[24] J. Wang, W. Yu, M. Gao, C. Gu, and Y. Yu, "Preoperative prophylactic intraaortic balloon pump reduces the incidence of postoperative acute kidney injury and short-term death of high-risk patients undergoing coronary artery bypass grafting: a meta-analysis of 17 studies," The Annals of Thoracic Surgery, vol. 101, no. 5, pp. 2007-2019, 2016.

[25] S. Wolfrum, K. S. Jensen, and J. K. Liao, "Endothelium-dependent effects of statins," Arteriosclerosis, Thrombosis, and Vascular Biology, vol. 23, no. 5, pp. 729-736, 2003.

[26] H. L. Lazar, Y. Bao, Y. Zhang, and S. A. Bernard, "Pretreatment with statins enhances myocardial protection during coronary revascularization," The Journal of Thoracic and Cardiovascular Surgery, vol. 125, no. 5, pp. 1037-1042, 2003.

[27] M. Haase, A. Haase-Fielitz, S. M. Bagshaw et al., "Phase II, randomized, controlled trial of high-dose $\mathrm{N}$-acetylcysteine in high-risk cardiac surgery patients," Critical Care Medicine, vol. 35, no. 5, pp. 1324-1331, 2007.

[28] K. Bando, K. Sun, R. S. Binford, and T. G. Sharp, "Determinants of longer duration of endotracheal intubation after adult cardiac operations," The Annals of Thoracic Surgery, vol. 63, no. 4, pp. 1026-1033, 1997.

[29] D. Taggart, "Respiratory dysfunction after cardiac surgery: effects of avoiding cardiopulmonary bypass and the use of bilateral internal mammary arteries," European Journal of Cardio-Thoracic Surgery, vol. 18, no. 1, pp. 31-37, 2000.

[30] C. M. Cox, R. Ascione, A. M. Cohen, I. M. Davies, I. G. Ryder, and G. D. Angelini, "Effect of cardiopulmonary bypass on pulmonary gas exchange: a prospective randomized study," The Annals of Thoracic Surgery, vol. 69, no. 1, pp. 140-145, 2000. 


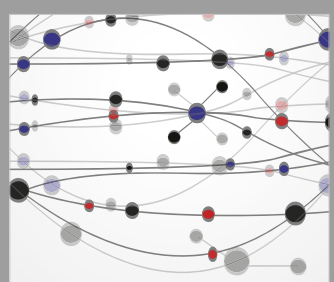

The Scientific World Journal
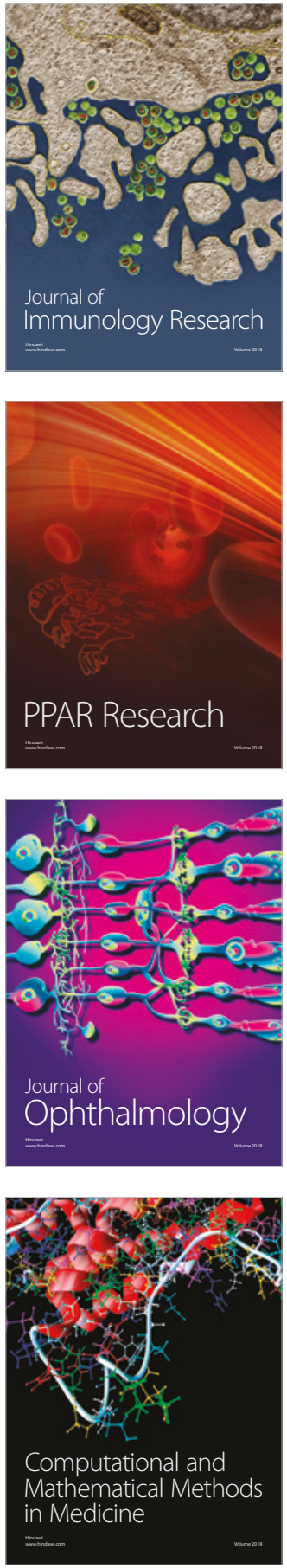

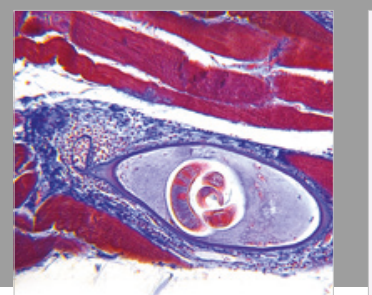

Gastroenterology Research and Practice

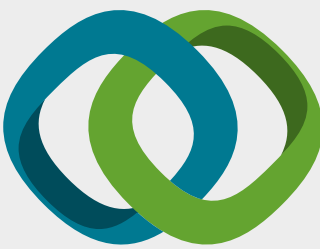

\section{Hindawi}

Submit your manuscripts at

www.hindawi.com
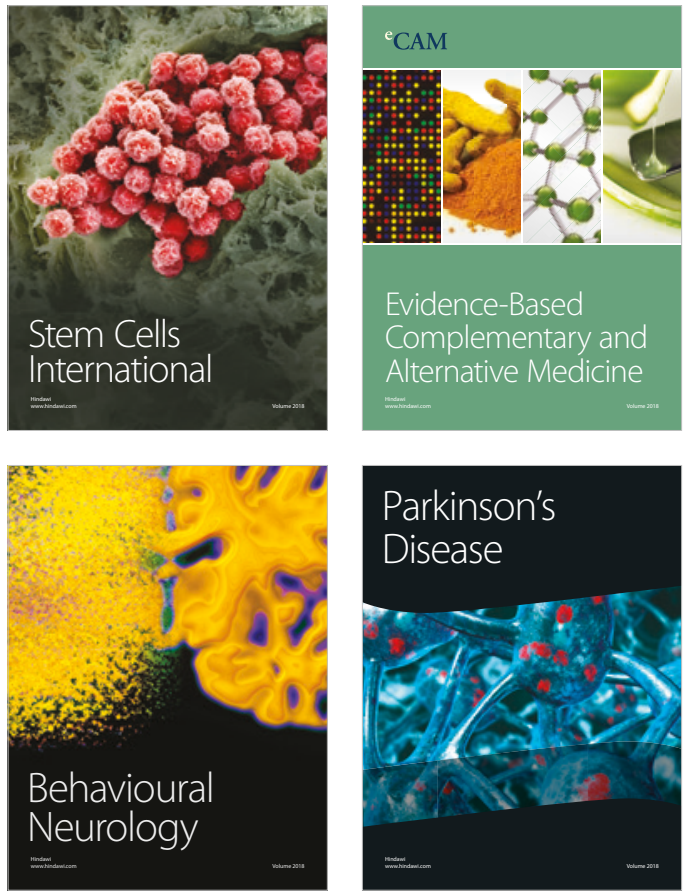

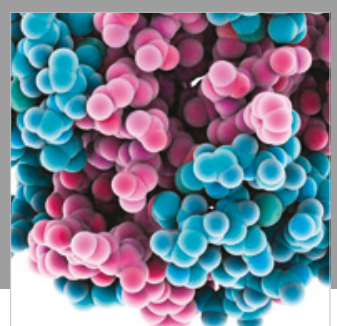

ournal of

Diabetes Research

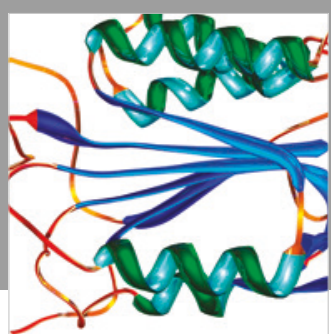

Disease Markers
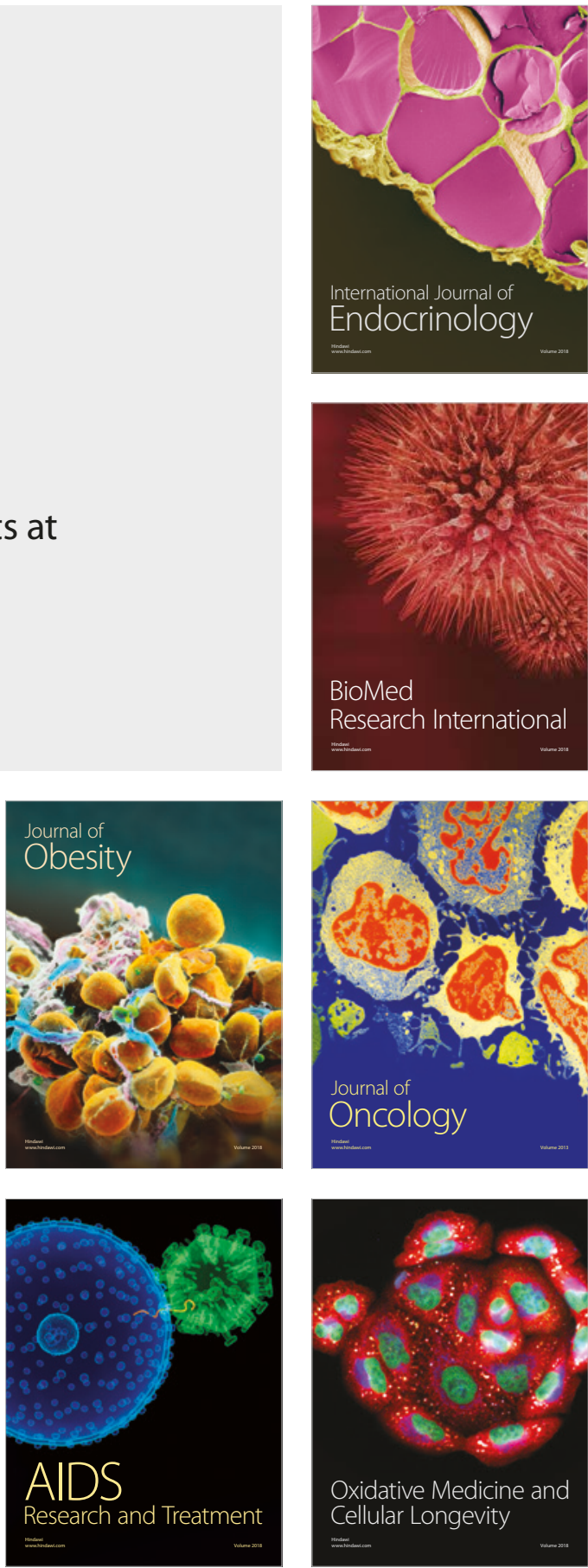